Journal of The Faculty of Science and Technology (JFST)

http://journal.oiu.edu.sd/index.php/JFST

https://doi.org/10.52981/jifst.vi7.951

ISSN: 1858 - 6007

$$
\text { مجلة العلوم و التقانة - جامعة أم درمان الإسلامية - وزارة التعليم العالي و البحث العلمي ـ السودان }
$$

\title{
Limit Cycle Problem for Quadratic System with some Applications of a Class I
}

\author{
Ali Bakur Barsham ALmurad ${ }^{1}$, Elamin Mohammed Saeed Ali ${ }^{2}$ \\ ${ }^{1}$ Department of Mathematics. Faculty of Education. AL-Salam University. \\ E.Mail: alibakur1976@gmail.com \\ ${ }^{2}$ Department of Mathematics. Faculty of Education. Alzaem Alazhari University.
}

\begin{abstract}
This paper is part of a wider study limit cycle problems of a class and planar system; we study the existence of limit cycle for polynomial planar system. In this paper, we present a proof of a result on the existence of limit cycle of the Quadratic System: $\frac{d x}{d t}=P_{2}(\mathrm{x}, \mathrm{y}), \frac{d y}{d t}=Q_{2}(\mathrm{x}, \mathrm{y})$
\end{abstract}

Key words: Quadratic system, Limit cycle, Quadratic polynomials.

\section{Introduction:}

We are interested in existence of limit cycle for some polynomial planar system. We discuss the existence by applying theorem 1 in [2]. We introduce the Quadratic system and give a Theorem 1 for existence of limit cycles. We apply Theorem 1 for existence of limit cycles for some applications and verify the exactions.

\section{The Existence of Limit Cycles:}

We consider the quadratic differential system

$\frac{d x}{d t}=P_{2}(\mathrm{x}, \mathrm{y}), \frac{d y}{d t}=Q_{2}(\mathrm{x}, \mathrm{y})$

Where $P_{2}$ and $Q_{2}$ are general quadratic polynomials.

In this case the system (1) under this transformation becomes

$$
\frac{d x^{l}}{d t}=P_{2}^{l}\left(\mathrm{x}^{l}, \mathrm{y}^{l}\right), \frac{d y^{l}}{d t}=\mathrm{x}^{l}\left(\mathrm{ax}^{l}+\mathrm{by}^{l}+\mathrm{c}\right)
$$

The system (2) has three classes 


$$
\mathrm{I}^{a}{ }^{a=b}=0, c \neq 0,
$$

$\frac{d x}{d t}=k+\delta x+e y+t x^{2}+\mathrm{mxy}+\mathrm{ny}^{2}, \frac{d y}{d t}=c x$

II. $a \neq 0, b=0, c \neq 0$,

$\frac{d x}{d t}=k+\delta x+e y+t x^{2}+\mathrm{mxy}+\mathrm{ny}^{2}$,

$\frac{d y}{d t}=x(\mathrm{a} x+c)$.

$b \neq 0$,

III.

$$
\frac{d x}{d t}=k+\delta x+e y+t x^{2}+\mathrm{mxy}+\mathrm{ny}^{2},
$$$$
\frac{d y}{d t}=x(\mathrm{a} x+b y+c) .
$$

To change (3- 5) into

I. $\frac{d x}{d t}=-y+\delta x+t x^{2}+\mathrm{mxy}+\mathrm{ny}^{2}, \frac{d y}{d t}=x$

II. $\frac{d x}{d t}=-y+\delta x+t x^{2}+m x y+\mathrm{ny}^{2}$,

$$
\frac{d y}{d t}=x(1+\mathrm{a} x), \mathrm{a} \neq 0 .
$$

III. $\frac{d x}{d t}=-y+\delta x+\imath x^{2}+\mathrm{mxy}+\mathrm{ny}^{2}$

$$
\frac{d y}{d t}=x(1+\mathrm{a} x+b y), \mathrm{b} \neq 0 .
$$

First studied necessary and sufficient conditions for the quadratic system of the form

$$
\begin{gathered}
\dot{x}=-y+a_{20} x^{2}+a_{11} x y+a_{02} y^{2}, \\
\dot{y}=x+b_{20} x^{2}+b_{11} x y+b_{02} y^{2}
\end{gathered}
$$

With $(0,0)$ as its $\mathrm{k}$ th-order fine focus $(\mathrm{k}=1,2,3)$ and then derived necessary and sufficient conditions for $(0,0)$ to be a center.

Theorem1: For system (6) introduces the quantities 
$W_{1}=A \alpha-B \beta$,

$W_{2}=[\beta(5 \mathrm{~A}-\beta)+\alpha(5 \mathrm{~B}-\alpha)] \gamma$,

$W_{3}=(A \beta+B \alpha) \gamma \delta$.

Where

$$
\begin{aligned}
& A=a_{20}+a_{02}, \quad B=b_{20}+b_{02}, \\
& \quad \alpha=a_{11}+2 b_{02}, \quad \beta=b_{11}+2 a_{20}, \\
& \delta=a^{2}{ }_{02}+b^{2}{ }_{20}+a_{02} A+b_{20} B, \\
& \gamma=b_{20} A^{3}-\left(a_{20}-b_{11}\right) A^{2} B+\left(b_{02}-a_{11}\right) A B^{2}-a_{02} B^{3} .
\end{aligned}
$$

Then the following assertions are true:

(a) $(0,0)$ is a $\mathrm{k}$ th - order fine focus $(\mathrm{k}=1,2,3)$ if and only if the following kth group of conditions holds:
(i)
$W_{1} \neq 0$
(ii) $W_{1}=0, W_{2} \neq 0$;
(iii)
$W_{1}=W_{2}=0, W_{3} \neq 0$

(b) The stability of the $\mathrm{k}$ th - order fine focus decided by the sign of ${ }^{W_{k}}$ : it is stable for $W_{k}<0$ and unstable for $W_{k}>0$.

(c) The origin is a center if and only if $W_{1}=W_{2}=W_{3}=0$.

In order to study the stability of $(0,0)$, when only ${ }^{W_{1}}=0$ we have to examine ${ }^{W_{2}}$, and when only $W_{1}=W_{2}=0$ we have to examine ${ }^{W_{3}}$. Hence if we use the quantities

$W_{1}=A \alpha-B \beta$,

$W_{2^{l}}=\left\{\begin{array}{l}(5 \mathrm{~A}-\beta) \beta \gamma, \text { when } A \neq 0, \\ (5 \mathrm{~B}-\alpha) \alpha \gamma, \text { when } B \neq 0, \\ 0, \text { when } A=B=0\end{array}\right\}$

$$
W_{3^{l}}=\left\{\begin{array}{l}
\mathrm{A} \beta \gamma \delta, \text { when } A \neq 0 \\
\mathrm{~B} \alpha \gamma \delta, \text { when } B \neq 0 \\
0, \text { when } A=B=0
\end{array}\right\} .
$$

To replace $W_{1}=W_{2}$, and $W_{3}$ in (7), then the conclusion of theorem (1) remains unchanged. 
Corollary. System (6) takes the origin as its center if and only if at least one of the following four groups of conditions holds:

1) $A=B=0$ (equivalent to $\lambda_{1}=\lambda_{3}-\lambda_{6}=0$ ).

2) $\alpha=\beta=0$ (equivalent to $\lambda_{1}=\lambda_{4}=\lambda_{5}=0$ ).

3) $A \alpha-B \beta=\gamma=0$ (equivalent to $\lambda_{1}=\lambda_{2}=\lambda_{5}=0$ ).

4) $5 A-\beta=5 B-\alpha=\delta=0$ (equivalent to $\lambda_{1}=\lambda_{5}=\lambda_{4}+5 \lambda_{3}-5 \lambda_{6}=\lambda_{3} \lambda_{6}-2 \lambda^{2}{ }_{6}-\lambda_{2}^{2}=0$ ) .

\section{Applications}

2.1. We considered the following Quadratic system

$$
\begin{gathered}
\dot{x}=-y+\left(a x+b x^{2}\right) \\
\dot{y}=-\left(x+b x^{2}+c x^{3}\right)
\end{gathered}
$$

For the quadratic system of the form

$$
\begin{gathered}
\dot{x}=-y+a_{10} x+a_{20} x^{2}+a_{01} y+a_{02} y^{2}+a_{11} x y \\
\dot{y}=x+b_{10} x+b_{20} x^{2}+b_{01} y+b_{02} y^{2}+b_{30} x^{3}+b_{11} x y
\end{gathered}
$$

For system (11): $a_{10=} a, a_{20}=b, a_{01}=-1, a_{02}=0, a_{11}=0$

$$
b_{01}=b_{02}=0, \quad b_{10}=-1, \quad b_{20}=-b, b_{30}=-c, \quad b_{11}=0
$$

And apply the system (8):

$$
\begin{aligned}
& A=a_{20}+a_{02}=b+0=b \\
& B=b_{20}+b_{02}=-b+0=-b \\
& \alpha=a_{11}+2 b_{02}=0+2(0)=0 \\
& \beta=b_{11}+2 a_{20}=0+2(b)=2 b \\
& \delta=a_{02}^{2}+b^{2}{ }_{20}+a_{02} A+b_{20} B=b^{2}+b^{2}=2 b^{2}, \\
& \qquad \gamma=\mathrm{b}_{20} A^{3}-\left(a_{20}-b_{11)} A^{2} B+\left(b_{02}-a_{11}\right) A B^{2}-a_{02} B^{3}\right. \\
& =(-b)(b)^{3}-(b)(-b)(b)^{2}=b^{4}-b^{4}=0 .
\end{aligned}
$$

In traduce the quantities. For system (7):

$$
\begin{gathered}
w_{1}=b(0)-(-b)(2 b)=2 b^{2} \\
w_{2}=[0(5 b)+(0)(-5 b)] \cdot 0=0 \\
w_{2}=0 \\
w_{3}=(b(0)+(-b)(0)) \cdot 0=0 \\
w_{1} \neq 0, \quad w_{2}=w_{3}=0
\end{gathered}
$$


Which is the order fine focus.

2.2. Now we consider the following Quadratic system

$$
\dot{x}=k+\delta x+e y+l x^{2}+m x y+n y^{2}
$$

$\dot{y}=x(c+a x+b y)$

Let $k=0, e=-1, c=1$

$$
\dot{x}=-y+\delta x+l x^{2}+m x y+n y^{2}
$$

$\dot{y}=x+a x^{2}+b x y$

When $=0$, the system (14) becomes

$$
\dot{x}=-y+\delta x+\left(x^{2}+m x y\right.
$$

$\dot{y}=x+a x^{2}+b x y$

The case: $a=b=0$

$$
\dot{x}=-y+\delta x+l x^{2}+m x y
$$

$\dot{y}=x$

Apply the system (12)

For system (16): $a_{10=} \delta, a_{20}=l, a_{01}=-1, a_{02}=0, a_{11}=m$

$$
b_{10}=1, b_{11}=b_{20}=b_{01}=b_{02}=0,
$$

And apply the system (8)

$$
\begin{aligned}
A & =a_{20}+a_{02}=l+0=l, \\
B & =b_{20}+b_{02}=0 \\
\alpha & =a_{11}+2 b_{02}=m, \\
\beta & =b_{11}+2 a_{20}=2 l, \\
\delta & =a^{2}{ }_{02}+b^{2}{ }_{20}+a_{02} A+b_{20} B=0, \\
\gamma & =\mathrm{b}_{20} A^{3}-\left(a_{20}-b_{11}\right) A^{2} B+\left(b_{02}-a_{11}\right) A B^{2}-a_{02} B^{3} \\
\gamma & =0 .
\end{aligned}
$$

In traduce the quantities. For system (7):

$$
\begin{gathered}
w_{1}=l \mathrm{~m}-0=l m \\
w_{2}=[(2 l)(5 l-2 l)+m(-m)] \cdot 0=0 \\
w_{3}=(l(2 l)+0(m)) \cdot 0=0 . \quad \\
w_{1} \neq 0, \quad w_{2}=w_{3}=0
\end{gathered}
$$

Which is the order fine focus.

When $=0$, the system (14) becomes 


$$
\begin{gathered}
\dot{x}=-y+\delta x+l x^{2}+n y^{2} \\
\dot{y}=x+a x^{2}+b x y
\end{gathered}
$$

The case: $a=b=0$

$$
\dot{x}=-y+\delta x+l x^{2}+n y^{2}
$$

$\dot{y}=x$

Apply the system (12)

For system (18): $a_{10=} \delta, a_{20}=l, a_{01}=-1, a_{02}=n, a_{11}=0$

$$
b_{10}=1, b_{11}=b_{20}=b_{01}=b_{02}=0 \text {, }
$$

And apply the system (8)

$$
\begin{aligned}
A & =a_{20}+a_{02}=l+n, \\
B & =b_{20}+b_{02}=0 \\
\alpha & =a_{11}+2 b_{02}=0 \\
\beta & =b_{11}+2 a_{20}=2 l, \\
\delta & =a_{02}^{2}+b^{2}{ }_{20}+a_{02} A+b_{20} B=n^{2}+n(l+n)=2 n^{2}+n l, \\
\gamma & =\mathrm{b}_{20} A^{3}-\left(a_{20}-b_{11}\right) A^{2} B+\left(b_{02}-a_{11}\right) A B^{2}-a_{02} B^{3} \\
\gamma & =0 .
\end{aligned}
$$

In traduce the quantities. For system (7):

$$
\begin{gathered}
w_{1}=(l+n) \cdot 0-0(2 l)=0 \\
w_{2}=[2 l(5(l+n)-2 l)+0] \cdot 0=0 \\
w_{2}=0 \\
\left.w_{3}=((l+n) 2 l+0)\right) \cdot 0=0 \\
w_{1}=w_{2}=w_{3}=0
\end{gathered}
$$

Which is the origin is a center.

\section{References:}

Butris R. N. (1991): Existence Of periodic solution for nonlinear systems of differential equations of operators with impulsive actions, Kiev, Ukraine Math, (joumal), Springer New York.

Cherkas and L. Zhilevich, Some criteria for the absence of limit cycle and for the existence of a single limit cycle, Differential Equations,(1977).

Cherkas, Estimation of the number of limit cycles of autonomous systems, Differential Equations, 13 (1977). 
Chicone and M. Jacobs, Bifurcation of limit cycles from quadratic isochrones, J. Dif. Eq. 91 (1991).

Ling, a concrete example of the existence of four limit cycles for plane quadratic systems, Sci. Sinica, (1980).

Llibre J. and Dana Schlomiuk, The geometry of quadratic differential systems with a weak focus of third order, Condition J. of Math, (2004).

Perko L., Differential Equations and Dynamical systems. Third Edition. Springer, New York, (2001).

Yan-Qian Y et al., Theory of Limit Cycles, Trans. Math. Mono, A.M. Soc. V 66, 1986. 